\title{
Moment Machine: Opportunities and Challenges of Posting Situated Snapshots onto Networked Public Displays
}

\author{
Nemanja Memarovic ${ }^{1}$, Ava Fatah gen Schieck ${ }^{2}$, Efstathia Kostopoulou ${ }^{2}$, \\ Moritz Behrens ${ }^{2}$, and Martin Traunmueller ${ }^{3}$ \\ ${ }^{1}$ Faculty of Informatics, University of Lugano (USI), Lugano, Switzerland \\ ${ }^{2}$ The Bartlett, University College London, London, UK \\ ${ }^{3}$ UCL ICRI-Cities, University College London, London, UK \\ nemanja.memarovic@usi.ch, \\ \{ava.fatah, ucftefk, moritz.behrens, \\ martin.traunmueller.11\}@ucl.ac.uk
}

\begin{abstract}
Large public displays are becoming a ubiquitous resource in the urban environment. Interconnected over the Internet these hitherto isolated "ad displays" could become a novel and powerful communication medium - networked public displays. One example for such a novel type of communication is their use as community tools. Scattered across the urban landscape and equipped with additional sensors, such as cameras, they provide the opportunity for local community members to take images of themselves and leave their "mark" in the setting, e.g., on their way to school, work, or meeting with friends. In order to understand the potential of posting situated snapshots on networked public displays in the context of place-based communities we designed and developed the Moment Machine - a networked public display application that allows one-click photo capture. In this paper we report on identified opportunities and challenges emerging from 6 user trials in the wild at 2 locations.
\end{abstract}

Keywords: networked public displays, urban screens, situated snapshots, community interaction, public space, urban computing, urban informatics.

\section{$1 \quad$ Introduction}

The urban landscape is getting "painted" with large public screens: from streets across small cafes to building facades we can find digital displays showing street maps, restaurant menus, or latest brand advertisement [10]. Although most of public displays represent singular installations that run locally stored power points or images, it is not hard to imagine that they will soon be connected and networked over the Internet [3, $15,16]$. Networked and empowered with additional sensors such as cameras networked public displays will constitute a novel and powerful communication medium for the $21^{\text {st }}$ century [3]. 
Dispersed across the urban landscape and embedded in the setting, they offer an opportunity to connect local community members, e.g., by having them support picture taking and sharing across the display network. For example, a school kid on his/hers way to school could be capturing images with his friends on a network of public screens, a group of teenagers could take photos while they are out-and-about on a Friday night, while adults with jobs could be taking photos to and from their way to work.

By taking images and leaving them in the settings (1) community members will express themselves and will leave their "mark". In return the photos taken by people in display locality could (2) stimulate community awareness by providing information on "who is around". They could also (3) enrich their locality and provide insights into other locations through photos captured in different environments, thus stimulating one of the human needs in public spaces: the need for discovery of new features and places [12].

As part of an ongoing "Screens in the Wild" research project [18] and in order to better understand the implications of posting situated snapshots onto networked public displays in the context of place-based communities we designed and developed Moment Machine - a networked public display application that allows one-click ondisplay photo capture. The Moment Machine is described in detail in section 3, right after the related work section (section 2). Our study design is summarized in section 4 where we describe six user trials "in the wild" at 2 locations where we conducted 55 interviews with passers-by. We present findings from our study in section 5, organized around (1) possible motivations for posting situated snapshots onto networked public displays, (2) discussion on where the images could be displayed once they are taken, and (3) challenges in promoting and sustaining their use. Finally we provide concluding remarks in section 6 .

\section{$2 \quad$ Related Work}

Research on networked public displays has seen its pioneering work in the early 80s where one of the prominent examples from that period is the 'Hole in Space' [5], a project that connected two urban spaces in New York and Los Angeles through a video link. Connected Urban Spaces [4] and Telectroscope [20] are examples of similar and more recent projects.

In more recent years work in the area has shifted from creating simple video links between two places into creating more interactive multimedia applications [2, 13] and how they can enrich urban spaces and promote community interaction and place awareness [11]. For example, Alt et al. [1] investigated how networked public displays can support exchange within urban communities by creating a digital version of traditional/analog notice boards.

The work of Ojala et al [16], Hosio et al. [6], North et al. [15], Peltonen et al. [17], and Taylor and Cheverst [19] falls closest to the work described in this paper as they have investigated how images can be used on networked public displays. Ojala et al.'s UBI-Postcard allows passers-by to take photos using a camera attached to a display. 
Once the photos are taken users can send the photo to an email address. Hosio et al.'s Ubinion similarly used public displays as input devices: young adults/teenagers could use them to create posters with speech bubbles that would represent their concerns about the city of Oulu. Posters created on displays (i.e., a network of displays) would be posted on dedicated Facebook and Twitter accounts. While UBI-Postcards and Ubinion used displays as input devices, Taylor and Cheverst's Wray Photo Display showed images uploaded from a dedicated website (images were uploaded by Wray villagers). Similarly, Peltonen et al.'s CityWall display showed user-contributed images from Flickr tagged with a specific tag. In the same manner North et al.'s ScreenGram shows images from Instagram with a predefined hashtag on a display network.

While all the above work used public displays for either taking the images $[6,16]$ or showing them on networked displays $[15,19]$ the study described here is the first one that used networked public displays for both. Also, our work complements the current body of research on networked public displays by (1) describing possible motivation for taking and posting situated snapshots onto public display networks, (2) discussing where could the images be displayed once they are taken, as well as (3) summarizing challenges in promoting and sustaining their use.

\section{The Moment Machine}

The Moment Machine is a networked public display application that allows passersby to simply capture their everyday moments by taking an image through a display and spreading it across the network. The Moment Machine's user interface is shown in Fig. 1-a. Its simple user interface was inspired by previous research that showed that passers-by do not spend a lot of time looking at public displays [7] and that live video feed represent a good mean for getting their attention [14]. For this reason we show a live video feed ( 1 in Fig. 1-a) and allow passers-by to take an image by simply pressing a button ( 3 in Fig. 1-a). Users can also change the "look" of their snapshot by selecting a filter before they take a photo ( 2 in Fig. 1-a), somewhat similar to the popular social media application Instagram [9]. In order to give the passers-by enough time to prepare themselves, image capture is delayed by five seconds, which is indicated through a countdown timer appearing instead of the "hands" button ( 2 in Fig. 1-a). Once the moment is captured users have thirty seconds to decide if they want to leave the moment on a display (shown in Fig. 1-b). After that the moment appears on the screen and across the display network (4 in Fig. 1-a). Passers-by also have the possibility to browse through moments captured at all locations (4 in Fig. 1-a).

\section{$4 \quad$ Study Design}

In order to uncover the potential of posting situated snapshots onto networked public displays we conducted six user trials "in the wild" at 2 locations in London, i.e., Walthamstow and Leytonstone. Both locations had a display in a public urban setting provided by the "Screens in the Wild" project [18] and were connected thus making a 
small display network ideal for technology probing [8]. User trials were conducted between 12AM and 5PM on working days. They lasted between 2.5 and 4.5 hours and partially overlapped. In total we conducted of 7.5 hours of trials at Leytonstone and 10.5 hours of trials at Walthamstow. Because the goal of the study was to "probe" and understand the implications of taking situated snapshots on local community members we decided to invite users to interact with the Moment Machine.
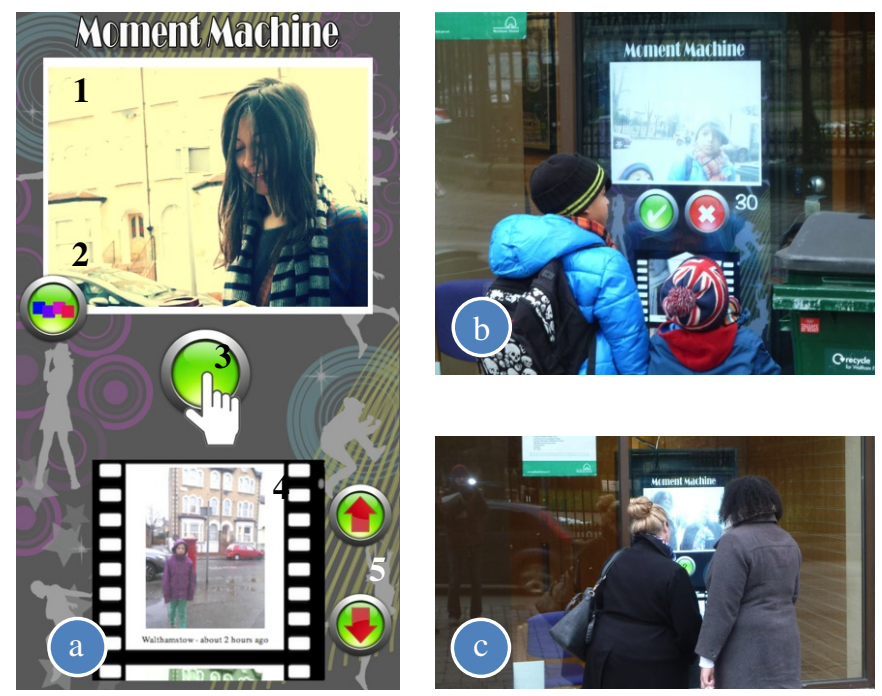

Fig. 1. The Moment Machine: a) user interface and b-c) passers-by interacting with it

During the trials we conducted 55 interviews (27 individual and 18 group interviews) with 71 people who interacted with the Moment Machine. At each location there were between 1 and 3 researchers who invited passers-by to interact with it. Passers-by were asked to take a photo and browse through existing ones on the display. After taking the snapshot researchers conducted semi-structured interviews and asked the participants about their general impression of the Moment Machine, e.g., would they come back to take more photos and browse through the ones shown on a display, and where they would like to see the images appear. After conducting the interview researchers wrote down the notes with some verbatim.

\section{$5 \quad$ Findings}

After collecting all the data we conducted an affinity diagram analysis and categorized the data, focusing on (1) possible motivations for taking images through networked public displays and leaving them in urban settings. We were also interested in (2) uncovering other digital places where the images could appear, e.g., Facebook or personal email as well as (3) understanding the challenges in promoting and sustaining the use of the Moment Machine application. 
Each interview received an identifier comprising from the first letter of the location ( $\mathrm{L}$ - Leytonstone, $\mathrm{W}$ - Walthamstow) and a sequential number of the interview, e.g., L01 would be the first interviewed person/group from Leytonstone while W04 would be the fourth interviewed person/group from Walthamstow. Considering the qualitative nature of our study our results are not intended as statistical evidence for expected behavior. Instead our results serve as an input to the design process by identifying potential tendencies of passers-by.

We first describe possible motivation for taking situated images and effects of posting them in urban settings, then we discuss where could the images taken through networked public displays appear, and in the end we present some of the challenges in sustaining their use.

\subsection{Motivations and Effects}

One of the possible reasons why passers-by would take images and leave them on public displays would be to "capture everyday life" (L04). For example, L06 stated that he would simply take pictures while going to or coming back from his work. Similar comment was received from L12 who said that he would take images on his way to and from school. Although we ran six user trials "in the wild" on arbitrary days this type of behavior was somewhat confirmed as we did get 3 returning users (L12, W18, W19) who brought additional participants to the display.

Taking images of oneself alone or in company of friends and family is another reason (W12, W13). This is also reflected with types of images people would like to see on public displays, i.e., images of people they know and have seen in their locality. As W12 points out "It is interesting if there is a photo of someone I know." As mentioned previously, the Moment Machine was tested in settings where displays were already running scheduled content. As interviewees from L01 point out "[we] will now pay more attention to the screen... [because we] expect to see images of people from Leytonstone [where they are residents] and our friends from Walthamstow." Similar comments were received from W31 who was commenting on people in the images "I know him... he lives there... he has a store..." Seeing images of people from the locality can bring up community awareness, i.e., who is around the neighborhood. This was explicitly stated by L05 and L13. However this type of communication can go even further and target individuals. For example, when capturing the moment W29 made a heart symbol with his hands. When asked why he did it he said that it is a sign between him and his girlfriend who was at work.

Another reason for taking the images would be getting " 5 minutes of fame". This is best captured by tongue-in-cheek shouting from two young girls "We are going to be famous!" Similarly, while a girl from L19 was taking a picture a man passing-by stated "Look, you're famous!" when he saw her picture on a display. Also, L16 stated that she likes that people can see her, while W17 was fascinated that people can see him at Leytonstone. Another benefit from capturing the moments outside would be to actually spend time in the physical environment. As L13 points out "Kids these days spend too much time in front of the computer, this might get them to play outside." 
Mashing of images/moments from the 2 locations can lead to universal connections, or "universality" as stated by L15. She liked the universality of the machine and that everyone can get connected and mashed through the Moment Machine.

In general people were interested in seeing images from the "other" location, thus reflecting the need for discovery of new features and places [12]. For example, the two people from L01 showed their interest in the Moment Machine application because they were from Leytonstone and had friends in Walthamstow. However, W11 from Walthamstow said that "[I] know the place [Leytonstone]. I don't know the people who live there." Because he did not know people from Leytonstone he was indifferent to seeing images from it. This shows that displays could connect to locations where users have friends/family/acquaintances, e.g., by harvesting a Facebook profile form users in the display vicinity.

While some people wanted to get more information from the other location, e.g., a live video feed (L09), others (W29) were concerned with this stating "I don't want just to spy on others through a website which connects me to cameras" and favored the asynchronous connection through images which allowed them to browse on their own.

\subsection{Where Should the Images Appear}

While most of the participants were comfortable to take the photo and leave it on displays, some wanted to know where will the image appear before taking the photo asking "Is it gonna stay here?" (L17, W14)

When we asked the participants where else would they like to see the images we received two types of answers: (1) either seeing them on a Facebook page, email, or dedicated website (18/26 participants) or (2) leaving the images to stay on the display (8/26 participants). Some of the participants did not see the point of leaving the image on the network unless they could "take" the moment with them. This is best captured by L14 who asked for her image to be removed since she could not take it with her. Interesting insight is also offered by L17 who wanted to get a printout of her photo this was preferred method over email. In essence this finding points out that networked public screens maybe need to be better connected with users "communicative ecology" [13], i.e., with current information and communication technologies such as Facebook and email.

On the other hand we also had participants who were satisfied with having the images appearing across the network, i.e., they did not want them to appear anywhere else: "absolutely not" stated L15 when asked if the images should appear somewhere else. She liked that the images are staying at the "machine" as they are "just capturing moments of everyday life."

\subsection{Challenges in Promoting and Sustaining Use}

One of the most prominent challenges in having people interested in interacting with the Moment Machine was scalability, i.e., the number of displays deployed in the urban space that run it (L07, W13, W34, W36). For example W13 points out that 
there are only two locations now that have this type of application while W36 stated that networked screens have to have a bigger scope and purpose.

Some of the challenges are posed by the urban environment that changes during the day. The research team noticed that when it was too bright it was hard to notice the video feed and participants had difficulties in understanding what is wrong and where they should position themselves to take the photo. Additional problems with positioning can be caused by camera placement. For example, some of the participants at Walthamstow had problems in positioning themselves to take a desired snapshot, e.g., a portrait. The camera used for photo taking was attached to the right side of the screen so if participants wanted to take a portrait they would have to look at the camera. However, some participants found this difficult and looked at the live video feed instead. Similar problems can be caused by the positioning of the screen itself. In order to make the user interface reachable to kids we intentionally made the buttons a bit lower. At Leytonstone the display is positioned a bit higher and both kids and adults did not report any problems interacting with it. However, W31 at Walthamstow commented that it is not comfortable to bend down in order to browse the photos.

Although the purpose of the application was clear to most of the participants, some had difficulties and questions regarding its purpose (L09, W26, W31, W34). A possible solution for this challenge would be to have a more obvious message on ta display, e.g., "Capture your daily moment" or "Strike a pose for the world!"

Finally some of the participants mentioned that the Moment Machine could benefit from adding new features (L09, W31, W33, W34). Some of the suggested features are relatively easy to add, e.g., adding the number of overall images that were taken (W31) while others are more sophisticated, e.g., adding image manipulation and ability to play with the background image (L09, W34). Adding new features could lead to users staying interested in the application, as suggested by Memarovic et al. [12].

\section{Conclusions}

In this study we used technology probe based approach to understand how posting situated snapshots on networked public displays could be beneficial for place-based communities. For this purpose we designed and developed the Moment Machine application and conducted 6 user trials "in the wild" at 2 locations in London. Our findings from observing 71 people interacting with the Moment Machine and conducting 55 interviews are promising and show that networked public displays can be used by local community members to capture images "on the go." In this paper we reported on (1) potential motivation for and effects of posting situated snapshots onto networked public displays, (2) where the images could be displayed once they are taken, and (3) challenges in promoting and sustaining their use.

Acknowledgments. The research leading to these results has received funding from the European Union Seventh Framework Programme (FP7/2007-2013) under grant agreement no. 244011, the EPSRC-funded project "Exploring the potential of networked urban screens for communities and culture" under the grant agreement no. EP/I031839/1, with the support of UCL VEIV center. We would also like to thank professor Marc Langheinrich for his useful comments on the early version of the paper and "Screens in the Wild" members for invaluable contribution. 


\section{References}

1. Alt, F., et al.: Digifieds: insights into deploying digital public notice areas in the wild. In: Proceedings of MUM 2011, pp. 165-174. ACM, New York (2011)

2. Clinch, S., Davies, N., Friday, A., Efstratiou, C.: Reflections on the long-term use of an experimental digital signage system. In: Proceedings of the 13th International Conference on Ubiquitous Computing, pp. 133-142. ACM, New York (2011)

3. Davies, N., Langheinrich, M., José, R., Schmidt, A.: Open Display Networks: A Communications Medium for the 21st Century. IEEE Compt. 45(5), 58-64 (2012)

4. Fatah gen. Schieck, A., Fan, S.: Connected urban spaces. In: Proceedings of SSS8 (2012)

5. Hole in Space, http: //bit. 1y/de6voG

6. Hosio, S., Kostakos, V., Kukka, H., Jurmu, M., Riekki, J., Ojala, T.: From School Food to Skate Parks in a Few Clicks: Using Public Displays to Bootstrap Civic Engagement of the Young. In: Kay, J., Lukowicz, P., Tokuda, H., Olivier, P., Krüger, A. (eds.) Pervasive 2012. LNCS, vol. 7319, pp. 425-442. Springer, Heidelberg (2012)

7. Huang, E.M., Koster, A., Borchers, J.: Overcoming assumptions and uncovering practices: When does the public really look at public displays? In: Indulska, J., Patterson, D.J., Rodden, T., Ott, M. (eds.) Pervasive 2008. LNCS, vol. 5013, pp. 228-243. Springer, Heidelberg (2008)

8. Hutchinson, H., et al.: Technology probes: inspiring design for and with families. In: Proceedings of CHI 2003, pp. 17-24. ACM, New York (2003)

9. Instagram, https://en.wikipedia.org/wiki/Instagram

10. Kostakos, V., Ojala, T.: Public Displays Invade Urban Spaces. IEEE Pervasive Computing 12(1), 8-13 (2013)

11. Memarovic, N., Langheinrich, M., Alt, F.: The interacting places framework. In: Proceedings of PerDis 2012. ACM, New York (2012)

12. Memarovic, N., Langheinrich, M., Alt, F., Elhart, I., Hosio, S., Rubegni, E.: Using Public Displays to Stimulate Passive Engagement, Active Engagement, and Discovery in Public Spaces. In: Proceedings of MAB 2012, pp. 55-64. ACM, New York (2012)

13. Memarovic, N., Langheinrich, M., Rubegni, E., David, A., Elhart, I.: Designing "interacting places" for a student community using a communicative ecology approach. In: Proceedings of MUM 2012. ACM, New York (2012)

14. Müller, J., Walter, R., Bailly, G., Nischt, M., Alt, F.: Looking glass. In: Proceedings of CHI 2012, pp. 297-306. ACM, New York (2012)

15. North, S., Schnädelbach, H., gen Schieck, A.F., Motta, W., Ye, L., Behrens, M., Kostopoulou, E.: Tension Space Analysis: Exploring Community Requirements for Networked Urban Screens. In: Kotzé, P., Marsden, G., Lindgaard, G., Wesson, J., Winckler, M. (eds.) INTERACT 2013, Part II. LNCS, vol. 8118, pp. 81-98. Springer, Heidelberg (2013)

16. Ojala, T., Kukka, H., Lindén, T., Heikkinen, T., Jurmu, M., Hosio, S., Kruger, F.: UBIhotspot 1.0. In: Proceedings of ICIW 2010, pp. 285-294. IEEE CPS (2010)

17. Peltonen, P., Salovaara, A., Jacucci, G., Ilmonen, T., Ardito, C., Saarikko, P., Batra, V.: Extending Large-Scale Event Participation with User-Created Mobile Media on a Public Display. In: Proceedings of MUM 2007, pp. 131-138. ACM, New York (2007)

18. Screens in the Wild, http: / / screensinthewild.org/

19. Taylor, N., Cheverst, K.: Supporting Community Awareness with Interactive Displays. IEEE Compt. (Special Issue on Interactive Digital Signage) 45(5), 26-32 (2012)

20. Telectroscope, http://www.telectroscope.net/ 